\title{
Seguridad Ciudadana: El Aporte de las Metodologías Implicativas
}

\section{Citizen Security: A contribution of Implacative and Participative Methodologies}

\author{
Mauricio García Ojeda ${ }^{1}$ \\ Alba Zambrano Constanzo ${ }^{2}$
}

\begin{abstract}
Resumen
El presente artículo revisa el concepto de seguridad ciudadana, proponiéndose que esta debe ser entendida en el contexto de los actuales procesos de exclusión social. Se expone que la gestión de la seguridad ciudadana que se realiza desde el ámbito público tiene a la base ciertas concepciones de esta, las que definen estilos de hacer que pueden orientarse hacia el cumplimiento de la normas y la vigilancia o tener a la base la valoración de participación de la ciudadanía.

En sintonía con este segundo estilo de abordar los problemas de seguridad ciudadana, se propone que esta debe concebirse desde una perspectiva amplia, fundamentada el ideario normativo del Desarrollo Humano y los derechos ciudadanos y que, por tanto, considere que las acciones emprendidas deben articularse integralmente, para que permitan superar condiciones de exclusión social. Para hacer viable lo anterior, es necesario que las diversas iniciativas de seguridad ciudadana operen desde el nivel local, con énfasis en el territorio y tendiendo a la base de las acciones emprendidas el uso de metodologías implicativas o participativas

Finalmente, se hace referencia a experiencias desarrolladas en España y en Chile, vinculadas a la seguridad ciudadana sobre la base de metodologías participativas. Además, se presentan reflexiones sobre el aporte de las metodologías implicativas para promover la ciudadanía y el desarrollo sustentable de las ciudades.

Palabras claves: Seguridad ciudadana, metodologías implicativas, exclusión social, desarrollo humano.
\end{abstract}

\section{Abstract}

This article reviews the concept of citizen security, proposing that this should be understood in the context of current processes of social exclusion. The management

1 Licenciado en Trabajo Social, Magíster en Desarrollo Humano a Escala Local y Regional. Estudiante del Programa de Doctorado en Sociología, opción Política Social, Universidad Autónoma de Barcelona. Investigador Asociado al Instituto de Desarrollo Local y Regional, Universidad de la Frontera. E - mail: garciam@ufro.cl

2 Psicóloga, Magíster en Ciencias Sociales Aplicadas, Maîtrisse en Ciencias de la Educación mención Desarrollo Social, ( ) doctor en Psicología Social. Docente del Departamento de Psicología, Universidad de La Frontera. E-mail: albaz@ufro.cl 
of citizen security that is carried out in the public sphere is based on certain conceptions, which define styles that can be oriented towards the fulfillment of norms and monitoring or be based on the valuation of citizen participation.

In consonance with this second style of confronting problems of citizen security, it is proposed that this should be conceived from a wide perspective, based on the normative principles of human development and citizen's rights and that, therefore, consider that actions undertaken should be integrally articulated, so that they may permit conditions of social exclusion to be solved. To make this viable, it is necessary that the diverse initiatives of citizen security operate from a local level, with emphasis on territory and having the use of implicative and participative methodologies at the base of the actions undertaken.

Finally, experiences in Spain and Chile are referenced, connected to the citizen security based on participatory methodologies. Also, reflections are presented on the contribution of implicative methodologies to promote citizenship and the sustainable development of cities.

Key words: citizen security, implicative methodologies, social exclusion, human development

\section{Introducción}

Por estos días, es fácil advertir en los discursos circulantes algunos eslóganes en torno a la seguridad ciudadana, una promesa fácil para una necesidad que parece crecer en el ambiente. Su uso y abuso cotidiano pareciera vaciar de contenido al concepto, simplificando en extremo un tema de alta complejidad.

Sin lugar a dudas, un rasgo común a las experiencias de la gente en las grandes ciudades de nuestro país, como en el resto del mundo, es el incremento de la violencia y la inseguridad. Pero pareciera ser que parte importante de las sensaciones de inseguridad, más allá de elementos concretos que parecen justificarla parcialmente, corresponden, en gran parte, al plano del imaginario del riesgo. Aquí, los medios de comunicación y el reordenamiento de los estilos de vida parecen jugar un papel fundamental (García, 2003).

La experiencia generalizada de inseguridad genera un predominio de lo privado sobre lo público, al refugio en los barrios cerrados por sobre la vida en espacios abiertos y compartidos. Con todo, la fragmentación y desconfianza social, parecen crecer a la par, aumentado aún más las sensaciones subjetivas de soledad, aislamiento e inseguridad.

Se invita a nuestros lectores y lectoras, a remirar en perspectiva la seguridad ciudadana como un logro compartido por los ciudadanos, como una construcción necesaria y urgente ligada estrechamente a la calidad de vida de las personas. Proponemos que esa construcción se vincula a la presencia de las democracias participativas, y a la gestión compartida de barrio y de ciudad. Aquí, son fundamentales las metodologías implicativas (o participativas) las que deben estar presentes en todas aquellas iniciativas que involucren "la vida en común" de las personas.

Una breve revisión conceptual nos permitirá resituarnos desde un pensamiento complejo, en una perspectiva más amplia del concepto, para luego revisar experiencias que intentan avanzar en la construcción de ciudades más seguras y sostenibles. Finalmente, se propone algunas reflexiones desde una vertiente metodológica respecto del diseño o rediseño de la dimensión relacional en el 
campo de la intervención psicosocial en el ámbito urbano.

\section{La seguridad ciudadana; evolución del concepto y contexto social actual en el que opera.}

La noción de seguridad ciudadana es amplia y polisémica. En términos generales se le puede vincular con otros conceptos que aluden a la idea opuesta, en algún sentido, a la idea de inseguridad. La teoría social ha desarrollado un amplio cuerpo de propuestas que coinciden en señalar que la actual sociedad moderna está cruzada por el riesgo. Si bien durante toda la evolución de la humanidad han existido condiciones de riesgo, nunca este ha alcanzado un nivel de complejidad y globalidad como el que actualmente experimentamos. El aspecto esencial que puede caracterizar el riesgo es que las personas tienen cada vez menos control directo sobre circunstancias que les pueden afectar negativamente (Bauman, 1996; Beck, 1998; Robles, 2000).

Es, por tanto, sintomático que en diversos niveles se definan normativamente variados aspectos vinculados con la seguridad; seguridad cooperativa, seguridad global, seguridad democrática, seguridad económica, seguridad alimentaria, seguridad ambiental y, como concepto amplio y abarcador, la seguridad humana (Ortiz, 2002). Un antecedente que permite dimensionar esta última es el Programa de Seguridad Humana aplicado en Canadá desde 1996, que incluye siete principios: 1) Seguridad económi$\mathrm{ca}$, la cual requiere un ingreso mínimo aceptable de "un trabajo productivo y remunerativo"; 2) Seguridad alimenticia, la cual se orienta a que "toda la gente en todo momento tenga acceso tanto material como económicamente a alimentos básicos"; 3) Seguridad de salud, especialmente para las clases menos favorecidas a nivel mundial; 4) Seguridad ambiental, definida como un medio ambiente saludable; 5) Seguridad personal, mediante la reducción de amenazas de tipo violento, criminal o de conflicto para cada individuo; 6) Seguridad comunal, esto es, "seguridad a través de la pertenencia a un grupo (siempre y cuando las normas sociales y las prácticas de comportamiento del grupo no amenacen la seguridad física del individuo como tal)"; 7) Seguridad política, "que toda la gente dentro de cualquier sociedad disfrute de todos sus derechos básicos» (PNUD, 1994, citado por Ortiz, 2002).

En el marco anterior, se pueden encontrar elementos que se vinculan a la seguridad ciudadana, especialmente en lo referido a la seguridad personal. Sin embargo, se aprecia que la noción de seguridad ciudadana se asocia actualmente a las acciones de cuidado de la propiedad privada y la integridad personal de las personas que habitan especialmente en ciudades. La seguridad ciudadana, por tanto, está cruzada por la noción de delito, por lo cual, la seguridad de los ciudadanos se hace posible a partir de la vigilancia y el resguardo físico y se hace énfasis en las "medidas de seguridad" para evitar delitos que infrinjan la ley y que dañen a las personas. Esta forma de concebir la seguridad ciudadana se ha convertido en un parámetro para evaluar la calidad de vida de los ciudadanos y a nivel estatal se definen políticas explícitas para su promoción.

Para comprender el actual alcance la seguridad ciudadana, se plantea que es necesario concebir la coevolución, por una parte de condiciones societales que configuran un marco de inseguridad y por otro, la concepción del ámbito público-estatal acerca de la seguridad ciudadana.

\section{a) Condiciones societales y seguridad ciudadana:}

La noción de seguridad ciudadana surge en el contexto de transformaciones en las sociedades contemporáneas. Se trata de un concepto asociado a los procesos de 
complejización social y heterogeneidad dinámica (cambios e interrelaciones desde el punto de vista productivo, de la estructura social, de las relaciones de familia, etnia y género, entre otras), en relación a lo cual se hace énfasis en la exclusión social. Se propone pasar del concepto restrictivo de pobreza al de exclusión social; el primero vinculado tradicionalmente a aspectos económicos (disponibilidad de ingresos monetarios) y el segundo expresado en diversas dimensiones; exclusión laboral, educativa, socio-sanitaria, urbana-territorial, familiar relacional, política, de ciudadanía y penal. La exclusión social se encuentra en la lógica analítica de la dinámica "dentro-fuera", que incluye conceptos como "marginación social”, "segregación", "aislamiento" y "desviación" (Tezanos, 1996). Específicamente, se concibe a la exclusión social como "la imposibilidad o dificultad intensa de acceder a los mecanismos de desarrollo personal e inserción socio-comunitaria y a los sistemas preestablecidos de protección" (Brugué, Gomá, Subirats, 2003: 3). La reducida disponibilidad de ingresos monetarios es un eje sustantivo de la exclusión social que gatilla otros tipos de exclusión. A nivel mundial, las desigualdades de ingresos han aumentado significativamente y en 1960, la diferencia entre el quintil con menos ingresos y el quintil con más ingresos era de 30 veces y en 1995 era de 80 veces (PNUD, 1998a). En el caso de Chile, si bien las políticas públicas, han logrado disminuir la pobreza y la extrema pobreza $^{3}$, la desigualdad en la distribución de los ingresos se ha mantenido prácticamente estable $^{4}$. En general, la exclusión social se expresa como un proceso estructural que tiene una dimensión global, vinculada a los procesos de polarización que generan desvinculación o desinserción social y una dimensión individual y familiar relacionada con las trayectorias que pueden direccionar vidas desde la integración a la exclusión. Además, existe una dimensión arriba-abajo (relativa a la pobreza) que se amplía a nivel societal con la distancia dentro-fuera. Robles (2000) se refiere a sociedades del riesgo residuales que son características de regiones de periferia moderna (capitalismo periférico), en donde existen «inseguridades manufacturadas» que afectan las biografías de los individuos y que, por tanto, modifican la interacción cotidiana. La individuación (radicalización insospechada e involuntaria de la individualización) caracteriza la exclusión masificada, que, para el caso de grupos, resulta ser una exclusión primaria (dificultad o no acceso a sistemas funcionales que pueden significar inclusión a prestaciones y servicios elementales). La individualización que erosiona la sociabilidad (Lechner, 2000), en contextos de exclusión, genera "trayectorias de inseguridad" (PNUD, 1998b), "atomización social" y un "efecto de precariedad" (Hopenhayn, 1994).

En las sociedades con crecientes tendencias dualizadoras, los sectores posicionados (estratos medios) y supraposicionados (estratos altos) se consideran, en la dicotomía den-

3 Las personas afectadas por la pobreza disminuyeron, durante la década de los noventa, de 5 a 3 millones, lo que significó una reducción en 10 años de un 39\% a un 21\% en relación al total de la población. La disminución de la extrema pobreza fue de un $7.3 \%$ (13\%, 1.600.000 personas a 5.7\%, 850.000 personas) (Hardy, 2002: 2; Castell, 2004: 142).

4 El coeficiente de Gini es de un 57.5 (el más alto de América Latina), lo que demuestra la permanencia de la desigualdad en Chile (Castell, 2004: 142). Además, Chile presenta la séptima peor distribución de los ingresos en el mundo (Meller, 2002: 14). Otros datos disponibles confirman esto; en 1987 el primer decil de ingreso autónomo (grupo más pobre) concentraba un 1.5\% de los ingresos y en 1996 el 1.4\%. Como contrapartida, para esos mismos años, el décimo decil concentraba un invariable el 41.3 (MIDEPLAN, citado por Baytelman, Cowan, De Gregorio, 1999:22). 
tro-fuera, como incluidos y los sectores infraposicionados (estratos bajos) están excluidos y las barreras de diferenciación entre incluidos y excluidos está representada por las oportunidades sociales (Tezanos, 1996).

Las masivas migraciones campo ciudad refuerzan las dinámicas de exclusión social. La atracción que generan los grandes centros urbanos y las mega-ciudades (Borja y Castell, 1997) hace que personas y familias de bajos ingresos se instalen en cordones marginales de las urbes y el propio entramado urbano de éstas, tiene como consecuencia que la exclusión tenga una expresión físico-espacial; se produce segregación espacial. Los hábitats residenciales son precarios, distantes y carentes de servicios sociales adecuados y son generadores de desintegración de los vínculos sociales.

El crecimiento estructural de la exclusión social -recordemos que los excluidos abarcan más de la mitad de la población de los países en el mundo- tiene una eficacia potente en quebrar la sociabilidad. Santos (2003), habla en este contexto del surgimiento de un "fascismo social", un conjunto diverso de relaciones sociales extremadamente desiguales en la propiedad del poder y capitales sociales. Ello implica que algunos (los que pueden tomar las decisiones), tienen derecho de veto sobre la vida de otros a pesar de que en lo formal se vive en sociedades democráticas.

Así, en sociedades socialmente fascistas aunque políticamente democráticas- la marginación y polarización consecuente generan ambientes propicios para la delincuencia y para la violencia, a la par que nuestros entornos de vida se hacen cada vez menos sustentables y habitables.

\section{b) La concepción del ámbito público- estatal acerca de la seguridad ciudadana:}

El concepto de seguridad ciudadana surge desde nociones vinculadas al orden pú- blico. Brotat (2002) señala que la idea de orden público en la tradición liberal está presente en la Declaración de los Derechos del Hombre y del Ciudadano promulgada en 1879, que expresa que en su artículo 9: “Nadie puede ser inquietado por sus opiniones, incluso por las religiosas, siempre y cuando su manifestación no altere el orden público establecido por ley". En el artículo 4 de esta declaración se indica que "La libertad consiste en hacer todo lo que no sea perjudicial al otro. Así, el ejercicio de los derechos naturales de cada hombre no tiene otro límite que aquellos que aseguren a los otros miembros de la sociedad el disfrute de estos derechos; estos límites sólo pueden estar determinados por ley". Para este autor el orden público se concibe como "garantía y límite de la libertad y, como ésta, consiste en que nadie puede hacer nada que pueda ser perjudicial a los demás". Por ello, las nociones de libertad y seguridad están vinculadas; "Sin seguridad no hay libertad (ya que la falta de ésta inquieta el libre ejercicio de los derechos y libertades del ciudadano) y sin libertad no puede haber una auténtica seguridad (ya que la seguridad sin seguridad comporta un régimen autoritario") (Brotat, 2002: 3-4). La seguridad y el orden social viabilizan la noción liberal de libertad que se concibe como libertad de: libertad de no verse impedido de hacer cualquier cosa que un individuo pueda querer hacer y además, libertad real para: libertad para que los individuos elijan entre las diversas vidas que pueden querer desarrollar (Van Parijs, 1996).

El concepto de orden público evolucionó paulatinamente hacia el de seguridad ciudadana. En forma posterior a la Declaración de los Derechos del Hombre y a los hechos de la Revolución Francesa, se generó un orden jurídico y político orientado a restaurar el antiguo régimen (Doménech, 2004) y en este marco, el Código Napoleónico y los marcos jurídicos inspirados en él, establecieron durante el siglo XIX y la primera mitad del siglo 
XX, un orden público en torno a la oposición orden-desorden, para lo cual se centró el énfasis en mantener la autoridad y perpetuar la norma y se tuvo como misión el forzar a los ciudadanos en la obediencia de la norma. Posteriormente, el orden público se asoció (con la inclusión de los valores del Estado social democrático y de derecho) a la seguridad ciudadana en forma explícita desde la segunda mitad del siglo XX en adelante. A partir de la dicotomía seguridad-inseguridad, los objetivos se orientaron a la preservación de derechos y libertades y el ofrecer un servicio público y la misión se inspiró en proteger la seguridad (objetiva y subjetiva) de los ciudadanos y en mejorar su calidad de vida. Actualmente el concepto de orden público (ordre public, public order o ordine pubblico) es utilizado en Europa como garantía de seguridad pública, pero con un sentido diferente, ya que se ha experimentado un tránsito desde la noción de forzar a los ciudadanos a que obedezcan una norma hacia la garantía de la calidad de vida de estos (Brotat, 2002).

En América Latina, el concepto de seguridad también estuvo vinculado al de orden público, pero en las décadas de 1970 y 1980, en el marco de la gestión de lo público que hicieron los diversos regímenes dictatoriales, se le concibió en el contexto de la doctrina de seguridad nacional, sobre todo en lo relacionado a la tesis del enemigo interno.

Actualmente, se otorga relevancia creciente a la seguridad ciudadana desde el modelo de seguridad denominado seguridad comunitaria en el que se incorpora al ciudadano en la formulación y ejecución de políticas de seguridad. Este modelo de seguridad se presenta como derivación de uno de los dos modelos policiales prototípicos; el inglés o de policía comunitaria, frente al francés o de policía del prínceps. El segundo, está vinculado a la construcción del Estado Nación Contemporáneo, por lo que se interesaba su defensa ante amenazas externas e internas. Este también llamado modelo latino, continental o napoleónico, operó a través de una estructura militar, jerárquica, centralizada y extendida por un territorio, como una policía represiva y reactiva al delito. El primer modelo, por su parte, la policía sería una consecuencia de las demandas sociales, operaría como una self-policy, una policía de carácter civil y local, preactiva y enfocada al servicio del cives, de la comunidad. Aquí los ciudadanos están interesados en colaborar con la actuación policial, pues les interesa la seguridad como un bien público (Brotat, 2002).

\section{Hacia un concepto inclusivo de seguridad ciudadana.}

Teniendo como antecedentes lo antes presentado, se postula que la noción de seguridad ciudadana debe contemplar el contexto societal en que se espera que su ideario normativo sea posible. Al respecto se coincide con lo señalado en el Manifiesto y las Resoluciones de los temas abordados por ciudades europeas sobre Prevención y Seguridad en la Conferencia de Nápoles en el 2000, citada por Brotat $(2002,8)$ : “La inseguridad no puede reducirse a los temas de criminalidad. La inseguridad es una problemática compleja, está atada a los problemas de sanidad, de medio ambiente, de urbanismo, de formación; es el resultado de desigualdades crecientes en el acceso a recursos; pone en juego conflictos de intereses, sobre todo con respecto a la división y al uso de espacios y de los ritmos de la ciudad (...). La inseguridad es un rasgo urbano al que hace falta darle respuestas civiles".

Se debe tener a la base de la noción de seguridad el concepto de complejidad de lo social, por tanto no son efectivas las soluciones simples. Los altos índices de violencia y criminalidad, no se reducirán sólo tematizando estas problemáticas en forma mediática y aumentando la vigilancia policial y la protección de los recintos privados. Es necesario generar logros en diversos niveles que permitan, por una parte, reducir los crecientes niveles de 
dualización y exclusión social y por otra parte, involucrar a los ciudadanos en las acciones vinculadas a su propia seguridad, lo que implica tener como elementos centrales la prevención del delito, la participación ciudadana y la inclusión social. Esta última, al decir de Yepes, (1998), debe concebirse como vinculada a la ciudadanía social y desde esta perspectiva la exclusión social puede ser observada como la negación de derechos sociales y oportunidades vitales fundamentales.

Según lo anterior, se concibe como pertinente la conceptualización de seguridad ciudadana propuesta en el Seminario sobre reflexión científica sobre el Delito y la Seguridad de los habitantes realizado en Costa Rica en 1995 (citado por Brotat, 2002:9): “(...) Un concepto verdaderamente amplio y comprensivo de la seguridad de los habitantes tiene que comprender no solamente la tranquilidad de no ser víctima de hechos delictivos, sino, también, la de vivir en un Estado Constitucional de Derecho y la de participar de los beneficios del desarrollo en materia de salud, educación, vivienda, ocio y todos los ámbitos del bienestar social. El concepto es el de Desarrollo Humano Sostenible, que tiene la equidad como principio". En esta misma línea el ya citado Manifiesto de Nápoles señala: "La seguridad es un bien común esencial para el desarrollo sostenible. Es a la vez signo y condición de inclusión social, del acceso justo a otros bienes comunes como son la educación, la justicia, la salud y la calidad del medio ambiente. Promover la seguridad es desarrollar un bien común y no consiste, sobre todo, en hacer policía o recurrir al sistema penal".

Vista así, la seguridad ciudadana, anclada en el concepto de Desarrollo Humano, (como ampliación de capacidades y oportunidades), puede concebirse de forma amplia como las condiciones que generan un contexto que minimiza los riesgos y potencia el tejido social para que las personas, en tanto ciudadanos sujetos de derechos, puedan desarrollarse de acuerdo a los proyectos de vida que han definido.

\section{La participación ciudadana y el enfoque territorial como claves para la seguridad ciudadana.}

El progresivo énfasis de políticas, planes y programa de seguridad ciudadana, tiene como un lugar común el que la participación ciudadana es necesaria. Sin embargo, es importante concretar los discursos y planteamientos genéricos con acciones efectivas e inclusivas. Esto implica un cambio en la cultura de gestión del ámbito público. Al respecto se constata la existencia de experiencias centradas en las actuales nociones de governance local, gobernación urbana y gobiernos de proximidad (Blanco y Gomá, 2002; Ibarra, Martí y Gomá, 2002; Subirats, 2001), que permiten generar acciones colectivas específicas que consideran la diversidad de las problemáticas vinculadas a la seguridad humana. Crecientemente ganan legitimidad las acciones de escala urbana y de barrio asociadas a una comunidad. La seguridad ciudadana, por lo tanto, además de estar vinculada al ideario del Desarrollo Humano, debe basarse en el concepto de territorio.

El territorio permite enfocar las políticas, planes y programas de seguridad ciudadanas, articulando el bienestar de las personas desde el particularismo-participación (Subirats, 2001). Una seguridad ciudadana promovida a escala humana y desde el territorio hace posible:

a) Abordar desde una óptica más precisa los problemas específicos considerando la variable socio-espacial. Existe una territorialidad que permitiría definir una «graduación tipológica de espacios». Esta graduación no se produce desde distinciones físicas, sino de la interacción de factores físicos con factores sociales. Los espacios apropiados y percibidos «con 
sentido» devienen en «lugares» a los que se adscriben distinciones que van desde lo público a lo privado (Greene, 1999). El lugar pasa a ser una relación dada entre espacio y conducta. Los significados atribuidos a un lugar no sólo son experimentados por sujetos aislados, sino que "tienden a organizarse en experiencias comunes (...) que influyen en las formas de pensar, actuar y sentir (...). Un lugar para vivir se vuelve una interpenetración socio física en la que el hablar y el habitar el medio físico y el medio social se entrecruzan de forma simultánea (...)".El complejo de lugares que se producen desde múltiples identificaciones, percepciones de espacios físicos configuran los llamados «hábitat residenciales». Estos hábitat incorporan en su conformación, además de los aspectos físicos, la trama de relaciones sociales, la identidad cultural y los sentimientos comunitarios que, en conjunto, configuran un determinado clima social que ejerce profundas influencias sobre el estilo y calidad de vida que allí genera. Todo hábitat residencial influye sobre el medio ambiente social que se manifiesta a través del grado de satisfacción de los residentes del lugar, sus sentimientos de identificación y arraigo, la imagen que se han formado de éste, el nivel de avenencia con los vecinos, la sociabilidad y la participación comunitaria (De La Puente, 1993:6).

b) Incorporar los aspectos subjetivos relacionados a la seguridad-inseguridad. Los diagnósticos vinculados a esto en las urbes, dan cuenta de la existencia de una "retracción de la sociabilidad", un "deterioro de lo público", un "atrincheramiento" de las personas en los ámbitos privados familiares, un creciente "nosotros restringido" y una debilidad del "nosotros colectivo", debido a la erosión de la confianza interpersonal y social que se expresa en un "miedo al otro" (PNUD, 1998b, 2000).

\section{Metodologías implicativas:}

Se ha propuesto hasta aquí que la única forma de hacer sustentable socialmente las ciudades -y con ello generar contextos socialmente seguros para los ciudadanos- es creando un clima de implicación social para resolver los problemas que afectan el buen vivir de la gente: el desempleo, la calidad de la educación, la salud, etc.

Hablamos de implicación para enfatizar la necesidad de "formar parte activa" de lo que colectivamente se construye, para diferenciar de lo que normalmente se viene entendiendo por participativo: incorporarse a la oferta que otros han definido.

Recordemos que la participación, en un sentido amplio, no sólo es un instrumento para la solución de problemas, como tiende a vérsele desde algunas perspectivas es sobre todo una necesidad fundamental del ser humano. Su práctica envuelve la satisfacción de otras necesidades básicas, como la interacción con otras personas, el desarrollo del pensamiento reflexivo, posibilidad de satisfacción de necesidades afectivas mediante la vinculación, autovaloración, posibilidad de expresarse y de crear (Arango, Marco, Castillo y Pavía, 1996).

La participación promueve el desarrollo personal y comunitario en la medida que favorece el desarrollo de diversas competencias, sentimientos de pertenencia, autonomía, proactividad, sentimiento de control psicológico y porque adicionalmente activa mecanismos que interconectan los espacios públicos y privados, haciendo que logro del bien común sea una tarea compartida.

Con esto queremos enfatizar que la participación, desde la perspectiva de la implicación, es fundamental para mejorar las condiciones de sociabilidad, para generar sentimientos de control y con ello de mejorar las condiciones de seguridad psicosocial. Pero además es la única forma, a nuestro 
entender, para encontrar soluciones creativas para los temas que ocupan a la gente.

En una mirada general, y como ya se ha indicado implícitamente, el concepto participación se tiende a usar en dos sentidos; uno el "ser participe de", es decir recibir prestaciones y disponer de servicios, y la otra "tomar parte de en", ello es la capacidad de desarrollar iniciativas, tomar decisiones, ser parte activa de un proceso.

La primera acepción entiende la participación como un elemento legitimador de poder, un mecanismo de integración; la segunda, en cambio entiende la participación como un elemento de transformación en el que todo el mundo tiene un papel protagonista. El comportamiento participativo desde esta posición sería "la acción o conjunto de acciones colectivas, acordadas reflexivamente y orientadas a la autogestión en la solución de los problemas y satisfacción de necesidades en donde se instauran principios de cooperación, solidaridad y ayuda mutua" (Arango, 1996:4).

Bajo esta comprensión, la participación sería un proceso activo y responsable de integración de actores diversos en una relación orientada al desarrollo de las personas que participan, y que se da en el encuentro de dos dinámicas: la capacidad de participar y la oportunidad de participar.

En este segundo significado la participación sería un proceso abierto, una construcción social que puede permitir realizar transformaciones en la realidad. Ello requiere sin duda, la creación de nuevas estructuras de relaciones que permitan que las decisiones acerca del futuro sean decisiones siempre compartidas (Rosa y Encina, 2004).

Este tipo de participación, va más allá de la información, de la asistencia a actividades que otros organizan o definen y trasciende la consulta de opinión acerca de determinadas cuestiones. Se trata, de acuerdo a lo se- ñalado por Villasante (2001) de avanzar hacia una ciudadanía con derecho a poder tomar parte de decisiones compartidas, después de un conocimiento de los problemas y alternativas.

En esta perspectiva, la participación permite a los individuos contrastar sus visiones acerca de la realidad junto a otros, creando visiones enriquecidas que mediante la interacción van permitiendo una plurideterminación de la realidad social. Los grupos sociales pueden así, tener su propia versión de la realidad situándose, entonces, de un modo distinto frente a la institucionalidad que normalmente impone la suya. En este sentido, la participación operaría como un mecanismo de redistribución de poder (Rosa y Encina, 2004).

Así entendida la participación, el tema de fondo es cómo generar procesos de esta naturaleza que permitan superar las resistencias que normalmente encontramos en los distintos ámbitos involucrados: la población, las administraciones políticas y los recursos técnicos y profesionales en juego.

Diversas experiencias a nivel territorial han centrado su énfasis en la participación de la comunidad como recurso esencial para la generación de condiciones de seguridad ciudadana sustentable. Al respecto, se citan iniciativas desarrolladas en España y Chile.

En el caso de España, destacan las diversas acciones generadas en diversas Comunidades Autónomas en las que existen comunidades y barrios marcados por la exclusión social, en donde se combinan las problemáticas que afectan tanto a españoles residentes como a la población migrante pobre que proviene desde otros países.

Son notables como ejemplos, primero, el Observatorio Participativo para la Convivencia Intercultural (OPCIIL) del barrio de Leganés. La percepción de los ciudadanos respecto a la seguridad se expresaba en fra- 
ses como "hay que ver, cada vez hay más inseguridad en las calles". El ayuntamiento comprobaba la limitada eficacia de sus acciones, principalmente debido a la descoordinación entre las instancias involucradas. Las preocupaciones centrales se centraban en las movilizaciones y conflictos surgidos en torno al debate sobre la Ley de Extranjería. Para abordar esta situación se creo un observatorio "desde el cual se puedan orientar las políticas municipales en materia de inmigración". A partir de la participación ciudadana se concretó un plan integral para idear el observatorio y en este proceso se integraron asociaciones de vecinos, Casas Regionales, sindicatos, asociaciones de corporación de la localidad, ONG's, organizaciones locales de inmigrantes, partidos políticos, representantes municipales, técnicos de diversas administraciones y vecinos en general (Martín, Montañez y Gutiérrez, 2002).

Un segundo ejemplo lo constituye el trabajo realizado desde la perspectiva de las redes participativas en el Programa de Participación Ciudadana en el Barrio del Pilar de Bailén, Jaén. En este barrio era necesario abordar problemas de marginación social. Un equipo técnico de los servicios sociales impulsó un Plan de Participación, en torno al cual se constituyó una Comisión de Seguimiento ciudadano que monitoreo el trabajo de cuatro grupos destinados a atender temas de urbanismo, salud, seguridad, educación y empleo (Martín, Montañez y Gutiérrez, 2002).

Un tercer ejemplo es el del Plan Comunitario y Social de la Trinitat Nova (PCTN) realizado en Barcelona. El barrio de Trinitat Nova situado en el distrito de Nou Barris, fue construido entre los años cincuenta y sesenta sin una planificación urbanística previa. Las viviendas y los servicios de urbanización eran precarios, los espacios públicos eran escasos y el sector estaba totalmente desconectado del núcleo urbano de la ciudad.
Se trata de un sector de residencia de mano de obra del sector industrial al que posteriormente llegaron oleadas sucesivas de inmigrantes extranjeros. En 1996, a partir del proceso de reflexión iniciada por la Asociación de Vecinos de la Trinitat Nova sobre la situación general del barrio, se define la necesidad de generar un plan comunitario para atender las condiciones de exclusión social existentes. Se generó un proceso de negociaciones que significó la existencia de apoyo institucional expresado en equipos técnicos y el financiamiento de iniciativas para el mejoramiento del barrio. En 1997, se firmó el Conveni de Barri por parte de la Direcció General de Serveis Comunitaris, el Districte Municipal de Nou Barri y la Asociación de Vecinos. El plan comunitario se concretó con diversas iniciativas que tuvieron como dimensiones de trabajo la urbanística, la educativa, la socio-cultural y la económica.

Finalmente, en el caso de Chile, además de las experiencias realizadas a partir de planes y programas de seguridad ciudadana ejecutados principalmente por municipios de la Región Metropolitana, se destaca la experiencia realizada por el Programa Chile Barrio a nivel nacional. Este programa surge en 1996 como una iniciativa interministerial destinada a la erradicación de los asentamientos precarios existentes en el país. Se definen como líneas de trabajo el Desarrollo Comunitario y la Inserción Social, el Desarrollo Laboral y Productivo y el Mejoramiento de la Vivienda y del Barrio. El trabajo con los pobladores y sus organizaciones se ideó una metodología participativa que incluyó la realización de diagnósticos participativos y la formulación, ejecución y evaluación participativa de planes de acción compartidos en los que, además de las familias de los asentamientos, se involucraron los municipios y otras organizaciones de nivel local y servicios públicos regionales. La experiencia ha permitido una mejora sustancial de la calidad de vida de un porcentaje significativo de las personas habitantes de 976 asentamientos precarios. La ac- 
ción sinérgica de diversos actores en varias dimensiones, tuvo consecuencias positivas en la seguridad ciudadana tanto en los nuevos barrios de viviendas sociales en los que habitan las personas como en las ciudades en las que estos conjuntos habitacionales se encuentran emplazados.

En otra experiencia desarrollada en la Comuna de Calama, Región de Antofagasta, denominada "Recuperación participativa de espacios públicos", se puede apreciar que la tarea de la seguridad ciudadana pasa por la recuperación y apropiación integral participativa de espacios que con anterioridad carecían de implementación comunitaria. Sobre la base del trabajo en torno a un objetivo común, recuperación y transformación de sitios eriazos considerados de alta peligrosidad, los pobladores inician un proceso de apropiación y transformación del espacio público.

La iniciativa se inscribe en una visión de la seguridad ciudadana "como una expresión del bienestar psicosocial de una comunidad" (Programa Ciudadanía y Gestión Local, 2003: 80), y por ello desde un enfoque preventivo se busca promover la integración social y la recuperación de vínculos que permiten soporte a las problemáticas sociales que derivan en situaciones de violencia o actos delictuales.

El epicentro de la acción práctica se localiza en la recuperación participativa de espacios en desuso y que representan una zona de potencial riesgo. Es una iniciativa de integración social, en la medida que la participación de diversos actores favorece la sociabilidad y la inclusión de ellos en las decisiones que permiten que se sientan parte de un sueño compartido y que se emprendan las acciones conjuntas que favorecen la confianza, la solidaridad y el sentido de pertenencia. También recupera desde la acción colectiva la dimensión ciudadana expresada en el derecho a vivir en un lugar digno y a co-responsabilizarse del propio hábitat.
La metodología de intervención implementada por la ONG SUR Profesionales, se caracteriza por la concertación de diversos actores públicos (vecinos, policía, autoridades, entre otros). La dimensión participativa -que es la que nos interesa destacar aquí- es entendida como la posibilidad de los actores de tomar decisiones. Son los propios pobladores quienes eligen en qué quieren transformar los espacios, debiendo organizarse para desarrollar las obras que han resuelto para ello. Adicionalmente, se trabaja con las autoridades y jefes de servicios para que ellos se hagan participes y apoyen las iniciativas de recuperación de 6 espacios públicos de la ciudad de Calama.

Como se puede apreciar, la iniciativa aporta en generar participación ciudadana, organización y articulación de actores locales en torno a un propósito compartido, que permite que los actores locales se corresponsabilicen de tareas que hacen posible mejorar sus condiciones de vida, todas ellas condiciones fundamentales para fortalecer la seguridad objetiva del entorno y la construcción de un imaginario de un espacio propio y compartido, bases de la seguridad subjetiva.

\section{El aporte de las metodologías implicativas}

En un contexto de creciente globalización y complejidad de las relaciones en diversos ámbitos, lo local recobra valor para generar procesos orientados al mejor vivir de la gente y a fortalecer las formas de convivencia y sociabilidad. Es sin duda en el ámbito local donde se pueden construir espacios para la concertación e implicación ciudadana entre los distintos sectores: político, económico, intelectual, profesional y organizaciones populares. En el territorio -socialmente construido- no sólo se concentra un grupo humano y una diversidad de actividades, sino que también constituye un espacio simbiótico que integra culturalmente (Borja y Castell, 1997:148-149). Entonces, el espacio de lo local puede ser un 
ámbito de respuestas posibles a los distintos desafíos, necesidades e intereses, que presentan las comunidades locales.

Las experiencias revisadas, dan cuenta que el estableciendo de consensos mínimos de múltiples actores permite construir soluciones en cuyo proceso se movilizan múltiples espacios que activan nuevas reflexiones e iniciativas. Es lo que Villasante (2001) denomina como "espiral creativa" en contraposición a los modelos cerrados que suelen estar detrás de las respuestas exclusivamente técnicas o políticas que predominan en el espacio comunal que a menudo no consideran o consideran muy parcialmente la participación de actores locales diversos.

Pero para lograrlo, la experiencia indica que se requiere de metodologías que colaboren en esta implicación social, que garanticen una relación entre sujetos (sujeto-sujeto), asegurando el protagonismo de las personas en situaciones que inviten a ser creativos porque los aportes de cada cual son realmente valorados. Se trata de propiciar distintos niveles de aprendizaje social compartido, que en una "espiral creativa" permitan readecuar actuaciones para ser cada vez más efectivos en una realidad social en permanente cambio.

Para que este proceso de creatividad social se construya requiere de metodologías también creativas, orientadas a activar la participación e implicación de los actores locales. Numerosas fuentes coinciden en que son las experiencias que se inscriben bajo el marco de la investigación-acción participante, con fuerte rigurosidad metodológica e inscritas en lógica de la complejidad (excluyendo aquellas orientadas exclusivamente por el voluntarismo) las que permiten llevar a la práctica lo que se ha logrado acordar colectivamente y adicionalmente reconducir las situaciones nuevas que se presenten, monitorear las dificultades no previstas, evaluando y reconstruyendo permanentemente el proceso.
A partir de lo antes expuesto, señalamos algunos criterios básicos para implementar estas metodologías, que siguiendo lo propuesto por Villasante, llamamos implicativas:

\section{Instalación de un proceso de transformación:}

Cuando hablamos de instalar un proceso de transformación, queremos hacer referencia a la dinamización que se debe realizar para que se produzcan condiciones para el encuentro, la reflexión, concertación de perspectiva, negociación y emprendimiento de acciones concretas, tareas todas desarrolladas en un contexto relacional que busca favorecer el respeto y la consolidación de estructuras democráticas.

Esa transformación se refiere a la mejora de condiciones, relaciones y recursos para la mejor vida de la gente. Esta transformación se fundamentaría en una visión integral e integradora de la comunidad (Rebollo, 2002). La visión integral alude a que tanto los problemas como los recursos presentes en ella, no pueden ser vistos de forma parcial o sectorializada. En una metodología comunitaria verdaderamente implicativa se debe planificar de abajo hacia arriba, ello con la finalidad de tener un visión multidimensional de los problemas y de las soluciones.

La visión integradora indica que en el proceso de transformación toda la comunidad puede y debe jugar un papel, no se trata de un plan para un determinado sector de la comunidad (sólo para pobres, sólo para jóvenes o sólo para mujeres). Se trata de un plan de y para toda la comunidad. Además, la visión integradora señala que el objetivo de la transformación debe ser siempre cohesionador e incluyente de la comunidad.

Se trata entonces, de favorecer un proceso -no cualquier proceso, sino uno de transformación de las relaciones y de las condiciones de vida- que amerita una mirada de 
conjunto y estratégica, que necesariamente debe ser construido participativamente por los distintos actores. Por lo tanto, no se trata de ejecutar proyectos para propósitos instrumentales específicos o fomentar la organización para acceder a recursos. Antes que nada se trata de poner en marcha un conjunto de relaciones que favorezcan la implicancia y la creatividad para ir tomando nuevas y más informadas decisiones acerca de un proyecto común.

\section{Concertación de actores diversos:}

Todo lo anterior plantea la necesidad de un trabajo que debe facilitar la participación en sus distintos niveles, asegurando mecanismos que mantengan informado del proceso al máximo de actores posibles, reforzando en las organizaciones sociales -que operan desde diferentes ópticas e intereses particulares- una visión de conjunto de lo que ocurre en el territorio.

Dado que las perspectivas, intereses y roles en juego son múltiples, es necesario conciliar la existencia de espacios específicos para cada bloque de actores locales. Las interrelaciones deben ser intencionadas sobre la base de intereses comunes, pero asumiendo que necesariamente el conflicto es parte de las relaciones y ellos bien resueltos pueden impulsar acuerdos de calidad.

A diferencia de los antiguos enfoques comunitarios que entendían por comunidad básicamente a la población, es necesario entender por comunidad a la globalidad de agentes y recursos políticos, técnicos y ciudadanos que habitan e intervienen sobre un territorio (Rebollo, 2002). Marchioni, destaca la necesidad de trabajar con los tres actores del proceso comunitario: ellos son: población, administración local y recursos técnico-profesionales. La relación dialéctica entre los tres protagonistas (o cuatro si agregamos a los agentes económicos) de los procesos y entre cada uno de ellos, ya que cada uno es una realidad heterogénea y compleja.

Como lo señaláramos con antelación, el trabajo con estos actores sociales que intervienen en el territorio se funda en la necesidad de que ellos vivan cambios en sus formas de actuar y de relacionarse habituales.

En síntesis, y en la perspectiva del trabajo en redes, se trata de conectar, coordinar y favorecer el trabajo en equipo de redes de actores que juegan roles complementarios en un territorio, transformando el estilo de relaciones para activar procesos de transformación social sustentables.

\section{Proceso diagnóstico y de planificación integral participativos:}

Esta interrelación y concertación de actores debe comenzar a propiciarse desde el momento en que se inicia el diagnóstico participativo, proceso crucial en cualquier iniciativa que busque con seriedad conseguir soluciones de fondo. El diagnóstico, debe entenderse primero que nada como un proceso de construcción de conocimiento y consensos acerca de la realidad que cada uno vive, eso significa reconocer problemas y recursos, la forma en que se relacionan las causas de los problemas, los roles que a cada uno y cada una le compete en esas soluciones. Un buen diagnóstico debe integrar múltiples niveles de información (cualitativa y cuantitativa), lo mismo que ser capaz de generar espacios que permitan encontrarse y debatir acerca de los temas que preocupan, en un proceso que en efecto espiral, vaya poniendo en interacción y vincule a nuevos actores (y redes de actores).

El conocimiento "producido", debe necesariamente sistematizarse y ponerse a disposición de la comunidad. Se trata de un diagnóstico vivo que va tomando fuerza en la medida que es compartido en su construcción mediante roles y tareas que variarán con 
los requerimientos de cada momento del proceso. La metodología de la investigación acción participante propuesta por autores latinoamericanos como Fals Borda (1998), y complementado con aportaciones europeas como las de Villasante (2001) y otros, resultan esenciales para fundamentar y orientar este tipo de diagnóstico.

Otra dimensión relevante es la elaboración de programaciones integrales, que aluden a la programación de acciones que buscan abordar los factores de fondo que se han identificado en relación a los problemas definidos. También en esta etapa se deben acordar qué entidades (actores) se harán cargo de qué acciones específicas, buscando mecanismos que garanticen la evaluación permanente y la retroalimentación de este conjunto de acciones emprendidas.

\section{Favorecer un proceso educativo transversal:}

Entendemos que el trabajo comunitario de corte implicativo, debe favorecer un proceso educativo a través de un trabajo humano y social que hace que la gente llegue a desarrollar su capacidad para interpretar la realidad y buscar formas de cómo cambiarla. Por lo tanto, este proceso social busca el cambio de actitudes, de valores que tienen que ser vividos y traducidos en nuevas formas de relación más democráticas. Ello demandará intencionar en la relación cotidiana espacios de reflexión sobre lo que se hace y se va viviendo, para recuperar el sentido pedagógico que los eventos cotidianos pueden tener. Los valores y las actitudes se concretizan a partir de nuestras acciones, de modo tal que es en las relaciones del cotidiano que se plasman nuestras contradicciones y aprendizajes. Esto requiere de una serie de acciones educativas en el cotidiano que permitan que cada uno de ellos en la interacción aprenda a relacionarse en la confianza, en la solidaridad en el respeto, en la colaboración.
En ocasiones también se requerirá de procesos más sistemáticos e intencionados sobre aspectos concretos que permitan tomar decisiones en mejores condiciones.

\section{Propiciar mecanismos de participación normalizados:}

La participación a pesar de sus múltiples beneficios, siempre supone un esfuerzo y costo para las personas, por ello se debe practicar formas de participación normalizadas, es decir que pueda practicar cualquier persona sin que su vida se vea alterada. La gente normal tiene que poder participar normalmente, siempre que pueda tener clara las finalidades y la globalidad del proceso y pueda colocar su cuota de participación (por poca que sea) en el contexto general de un proyecto más amplio y con sentido compartido.

Si la gente participa, significa que la gente puede participar dentro de su papel hasta el máximo de nivel posible de la participación que es poder co-participar o participar en las decisiones. Para ello se tienen que construir espacios colectivos que permitan que este momento decisional sea en referencia a la necesidad de la comunidad, resultante de una exigencia colectiva y no una cuestión personal como suele expresarse en el cacicazgo o el clientelismo en donde una persona maneja situaciones locales para fines particulares.

\section{Bibliografia}

Arango C., Marco, I., Castillo, R., Marco, C. \& PAVÍA, J. (1996). Identidad y participación en un barrio deprimido. En: A. Sánchez \& G. Musitu (Ed.) Intervención comunitaria: aspectos científicos, técnicos y valorativos. Barcelona: Universidad de Barcelona.

Arango, C. (1996). El comportamiento participativo en la investigación-acción. 
En: A. Sánchez \& G. Musitu (Ed.) Intervención comunitaria: aspectos científicos, técnicos y valorativos. Barcelona: Universidad de Barcelona.

BAuman, Z. (1996). Modernidad y Ambivalencia. En: J. Beriain (Comp.). Las consecuencias perversas de la modernidad. Barcelona: Anthropos.

Baytelman, Y., Cowan, K., \& De Gregorio, J., (1999). Política económico social y bienestar: el caso de Chile. Serie Económica, 56. Centro de Economía Aplicada, Departamento de Ingeniería Industrial, Facultad de Ciencias Físicas y Matemáticas, Universidad de Chile

BЕCK, U. (1998). La sociedad del riesgo: hacia una nueva modernidad. Barcelona: Paidós.

Blanco, I. \& Gomá, R. (2002). Gobiernos Locales y Redes Participativas. Barcelona: Ariel.

Borja, J. \& CASTELL, M. (1997). Local y global. La gestión de las ciudades en la era de la información. Madrid: Taurus.

BRotAT, R. (2002). Un concepto de seguridad ciudadana. http: / / www.dip-alicante.es / formacion / es / menu / almacen / BROTAT_seguridad_ciudadana. Consulta: Agosto de 2004.

Brugué, Q. Gomá, R. \& Subirats, J. (2003). De la pobreza a la exclusión social. Nuevos retos para las políticas públicas. Documento de trabajo, Institut de Govern i Polítiques Públiques: Universidad Autónoma de Barcelona.

Castell, M. (2004). Estado y Sociedad en la democracia chilena. Política Exterior. 100; 137-151.

De la Puente, P. (1993). Familia, vecindario y comunidad: un modelo sistémico para la interpretación del desarrollo progresivo. Estudios Sociales, 76; 149-167.
DOMÉnech, A. (2004). El eclipse de la fraternidad. Una revisión republicana de la tradición socialista. Barcelona. Crítica.

Equipo Académico del Programa Ciudadanía y Gestión Local (2003). Caminos de Innovación Ciudadana III. Resumen de las 30 experiencias finalistas del III Ciclo de premiación del Programa Ciudadanía y Gestión Local. Documento de Trabajo 11. Santiago de Chile.

Fals Borda, O. (1998). Participación popular: retos del futuro. Registro del Congreso Mundial de convergencia en Investigación Participativa'97: Estado del arte. Bogotá: ICFES.

García, N. (2003). Ciudades paranoicas miradas desde América Latina. En: Vanguardia Dossier. América Latina Democracia, Neoliberalismo, Populismo. 4; 46-52.

Greene, M. (1999). Vulnerabilidad al delito en el espacios residencial: un enfoque configuracional. En: DITEC-MINVU, Espacio Urbano, Vivienda y Seguridad Ciudadana. Documento de Colección Monografías y Ensayos, Serie VII.

HARDy, C. (2002). Resultados Encuesta CASEN 2000. Foro, Fundación Chile 21. www.fundacionchile21.cl. Consulta: Agosto de 2004.

Hopenhayn, M. (1994). Ni apocalípticos ni integrados. Aventuras de la Modernidad en América Latina. Santiago: Fondo de Cultura Económica.

IbARra, P., Martí, S. \& Gomá, R. (2002). Creadores de democracia radical. Movimientos sociales y redes de políticas públicas. Barcelona: Icaria.

LeCHNER, N. (2000). Desafíos de un Desarrollo Humano: individualización y capital social. Revista Instituciones y Desarrollo. 7; 7-34. 
Martín, P., Montañez, M., Gutiérrez, V. (2002). "La planificación comunitaria. Teoría y experiencias", en Ismael Blanco y Ricard Gomá (Coord.), Gobiernos Locales y Redes Participativas. Barcelona: Ariel.

Meller, P. (2002). Pobreza y distribución del ingreso en Chile (Década del 90). Documento de trabajo CIEPLAN.

Ortiz, J. (2002). La doctrina de la seguridad humana en la política exterior canadiense. La contribución de Lloyd Axworthy al estudio de la política mundial. Revista CIBOB d'Afers Internacionals. 60.

PNUD (1998a). Informe sobre Desarrollo Humano, Consumo para el Desarrollo Humano. Madrid: Mundi Prensa. (1998b). Desarrollo Humano en Chile 1998. Las paradojas de la Modernización. Santiago de Chile. (2000). Desarrollo Humano en Chile 2000. Más sociedad para gobernar. Santiago de Chile.

Programa Ciudadanía y Gestión Local (2003). Ampliando ciudadanía, promoviendo participación: 30 innovaciones locales. Santiago: Instituto de Estudios Públicos y Fundación Nacional para la Superación de la Pobreza.

Rebollo, O. (2002). Metodologías y prácticas transformadoras. El plan comunitario de Trinitat Nova. Barcelona.

Robles, F. (2000). El desaliento inesperado de la modernidad. Molestias, irritaciones y frutos amargos de la sociedad del riesgo. Santiago de Chile: RIL - Ediciones Sociedad Hoy.

Rosa, P. \& Encina, N. (2004). Democracias participativas desde las praxis locales. En: J. Encina, M. Rosa, A. Avila \& M. Fernández (Ed.). Democracias participativas e intervención social comunitaria desde Andalucía. Cuadernos de Ciudadanía. 5
SANTOS, B. (2003). La caída del angelous novas. Ensayos para una nueva teoría social y una nueva práctica política. Bogotá: Publicaciones ILSA.

SubIRATS, J. (2001). Educación y Gobierno Local. Barcelona: ARIBL.

Tezanos, J. (1996). Introducción. Tendencias de dualización y exclusión social en las sociedades tecnológicas avanzadas. Un marco para el análisis. Revista de Estudios Políticos. 91; 83-97.

VAN PARIJS, P. (1996). Libertad real para todos. Qué puede justificar el capitalismo (si hay algo que pueda hacerlo). Barcelona: Paidós.

Villasante, T (2001). Procesos para la creatividad social. En: T. Villasante, M. Montañés, \& P. Martín (Coord.). Prácticas Locales de creatividad social. Construyendo ciudadanía 2. Madrid: Viejo Topo.

YePES, I. (1998). El estudio comparativo de la exclusión social: consideraciones a partir de los casos de Francia y Bélgica. Revista Internacional del Trabajo. 113 (5-6); 669-721.

Fecha Recepción Artículo: 28 de junio 2005

Fecha Evaluación Final: 10 de abril 2006 